\title{
MOBS AND SEMITREES
}

\section{T. B. MUENZENBERGER and R. E. SMITHSON}

(Received 4 February 1974)

Communicated by $G$. E. Hall

In (1957) Ward characterized trees as being certain compact idempotent commutative monotone mobs. The purpose of the present note is to obtain a similar characterization for the semitrees as studied by Muenzenberger and Smithson $(1973,1975)$.

A $m o b$ is an associative semigroup together with a Hausdorff topology for which the multiplication is continuous. A mob $X$ is idempotent just in case $x x=x$ for all $x \in X$. Moreover, $X$ is monotone if and only if the set $\{(a, b): a b=x\}$ is connected in $X \times X$ for all $x \in X$. In other words, multiplication is a monotone function.

A semilattice is a partially ordered set $(X, \leqq)$ such that every pair of points $x, y \in X$ has an infimum $x \wedge y$ in $X$. Let $X$ be an idempotent commutative mob (sometimes called a topological semilattice). We endow $X$ with the relation $\leqq$ defined by: $x \leqq y$ if and only if $x y=x$ where $x, y \in X$. Clearly, $x \wedge y=x y$ for all $x, y \in X$. Ward (1957) showed that $\leqq$ is a partial order and that $L(x)=\{y: y \leqq x\}$ and $M(x)=\{y: x \leqq y\}$ are closed for all $x \in X$. He also showed that $\leqq$ is order dense in case $X$ is monotone. We shall use the following condition of Ward (1957).

CONDITION 0. Let $a, b, x \in X$. If $a x=a$ and $b x=b$, then either $a b=a$ or $a b=b$.

Observe that condition 0 is equivalent to assuming that $L(x)$ is a chain for all $x \in X$. A proof of the first lemma was given in the proof of Theorem 5 in Ward (1957), but the assumption of compactness is not needed. We give a slightly different proof.

LEMMA 1. If $X$ is an idempotent commutative mob that satisfies condition 0 , then $M(x)-\{x\}$ is open for all $x \in X$. 
Proof. Let $x \in X$ and let $\left\{x_{\alpha}\right\}$ be a net in $X$ that converges to $y \in M(x)-$ $\{x\}$. Then $x_{\alpha} y \in L(y) \cap L\left(x_{\alpha}\right)$ since $X$ is idempotent, and either $x_{\alpha} y \leqq x$ or $x \leqq x_{\alpha} y$ since $L(y)$ is a chain. If $x_{\alpha} y \leqq x$ for all $\alpha$, then $x_{\alpha} y \rightarrow y y=y \in L(x)$ since $L(x)$ is closed. Thus $x<x_{\alpha} y$ for some $\alpha$, and so $\left\{x_{\alpha}\right\}$ meets $M(x)-\{x\}$ which implies that $M(x)-\{x\}$ is open.

A portion of the next lemma also appears in Ward (1957).

Lемма 2. If $X$ is an idempotent commutative monotone mob, then $M(x)$ is connected for all $x \in X$. If, in addition, $X$ satisfies condition 0 , then every nonempty subset of $X$ that is bounded above has a supremum. In this case, $X$ is conditionally complete.

Proof. Let $m$ be the multiplication and let $\pi$ be projection onto the first coordinate. If $x \in X$, then $M(x) \subset \pi m^{-1}(x)$ since $M(x) \times\{x\} \subset m^{-1}(x)$. On the other hand, $\pi m^{-1}(x) \subset M(x)$ since $y z=x$ implies $y, z \in M(x)$. Thus $M(x)=$ $\pi m^{-1}(x)$ is connected for each $x \in X$.

Next let $A$ be a nonempty subset of $X$ and suppose that $B=$ $\cap\{M(a): a \in A\} \neq \varnothing$. Note that $B$ is closed. Also $b, b^{\prime} \in B$ implies $b b^{\prime} \in B$ and $b b^{\prime} \leqq b, b^{\prime}$. Assume that $\sup A$ does not exist. Then for all $b \in B$ there would be $b^{\prime} \in B$ such that $b^{\prime}<b$. It would follow that $B=$ $\cup\{M(b)-\{b\}: b \in B\}$ is open by Lemma 1 . Thus $B$ is a clopen set properly contained in $M(a)$ where $a \in A$, whereas $M(a)$ is connected. Consequently, sup $A$ must exist.

The authors are indebted to the referee for deleting a superfluous hypothesis in the following theorem and for providing the elegant proof presented below.

THEOREM 3. If $X$ is an idempotent commutative monotone mob, then $L(x)$ is connected for all $x \in X$.

Proof. Let $x \in X$. Then $X=\cup\{M(y): y \in L(x)\}$. Each $M(y)$ is connected by Lemma 2 , and so $X$ is connected since $x \in M(y)$ for each $y \in L(x)$. Therefore, $L(x)=X x$ is connected since multiplication is continuous.

Let $(X, \leqq)$ be a partially ordered set. If $x, y \in X$ and $x \leqq y$, then we define $[x, y]=M(x) \cap L(y)=\{z: x \leqq z \leqq y\}$,

$$
[x, y)=\{z: x \leqq z<y\},(x, y]=\{z: x<z \leqq y\}
$$

and $(x, y)=\{: x<z<y\}$.

In order to obtain the desired connection between mobs and semitrees, we need to obtain conditions under which $L(x)$ is compact.

THEOREM 4. Let $X$ be an idempotent commutative monotone mob that satisfies condition 0 and has a zero. Then $L(x)$ is compact for all $x \in X$ if and only if inf $A, \sup A \in A^{*}$ whenever $A \subset X$ is nonempty and bounded above. 
Proof. Suppose first that $L(x)$ is compact for all $x \in X$. In this case, the assumption that $X$ has a zero is redundant since Ward (1957) showed that $L(x)$ compact for some $x \in X$ implies that $X$ has a zero. Let $A \subset X$ be nonempty and bounded above. Suppose that $s=\sup A \notin A^{*}$. Then there exists an open set $U$ containing $s$ such that $U \cap A=\varnothing$, and

$$
\mathscr{U}=\{L(s)-M(a): a \in A\} \cup\{U\}
$$

would be an open cover of $L(s)$ with no finite subcover. Thus $s \in A^{*}$, and similarly inf $A \in A^{*}$.

To prove the converse, let 0 be the zero in $X$ and let $x \in X$. Then $L(x)=[0, x]$ is an order complete chain. Clearly, the relative topology on $L(x)$ contains the order topology. So we need only prove that the relative topology is contained in the order topology. For this purpose, let $y \in U$, a relatively open subset of $L(x)$. Suppose first that $y \neq 0$. If $U$ does not contain a set of the form $(a, y]$ where $0 \leqq a<y$, then for each $a \in[0, y)$ there would be $y_{a}$ such that $a<y_{a}<y$ and $y_{a} \notin U$. But then $\varnothing \neq A=\left\{y_{a}: a \in[0, y)\right\} \subset L(x)$ and $y=\sup A$, whereas $y \notin A^{*}$ since $U \cap A=\varnothing$. Thus $U$ must contain a set of the form $(a, y]$ for some $a \in[0, y)$. On the other hand, an analogous argument shows that $U$ contains a set of the form $[y, b)$ for some $b \in(y, x]$, if $y \neq x$. It now easily follows that $U$ is open in the order topology, completing the proof of the theorem.

Observe from the proof that $L(x)$ compact for $x \in X$ implies that the relative and order topologies on $L(x)$ coincide, and thus $L(x)$ must also be connected (being order complete and dense) and locally connected.

We now give some examples to show the significance of the conditions we have been using. Other pertinent examples will be presented later.

Examples. (1) Let $X=[0,1] \times[0,1]$ and set $(x, y) \cdot\left(x^{\prime}, y^{\prime}\right)=$ $\left(x \wedge x^{\prime}, y \wedge y^{\prime}\right)$ where $\wedge$ is the usual infimum in [0,1]. With the Euclidean topology, $X$ is a compact idempotent commutative monotone mob. But $X$ does not satisfy condition 0 , and there is $x \in X$ such that $M(x)-\{x\}$ is not open. Observe that $X-\{(0,1)\}$ is a mob that contains a chain with no supremum.

(2) Let $X=[0,1]$ and define $x y=x \wedge y$. We enlarge the usual topology on $X$ by declaring open sets of the form

$$
(1-\varepsilon, 1]-\{1-1 / n: n=1,2, \cdots\}
$$

where $\varepsilon>0$. With this enlarged topology, $X$ is an idempotent commutative monotone mob that satisfies condition 0 and has a zero, but $X$ contains a chain $A$ such that sup $A \notin A^{*}$. Consequently, $X$ is not compact.

(3) For $n=1,2, \cdots$, erect at $(1 / n, 0)$ in the plane a perpendicular interval of length $1 / n$. Let $X$ denote the union of the erected intervals and the unit interval. We enlarge the Euclidean topology on $X$ by requiring that the set 
consisting of the origin and everything above the $x$ axis be open. Now $X$ admits the structure of a complete semilattice - see Muenzenberger and Smithson (1973) - in which $M(x)$ is connected, $M(x)-\{x\}$ is open, and $L(x)$ is a chain which need not be connected for each $x \in X$. Here $\wedge$ is not continuous. A simple extension of this example produces a set $Y$ and two topologies $\mathscr{T}_{1}, \mathscr{T}_{2}$ for $Y$ such that $\left(Y, \mathscr{T}_{1}\right)$ and $\left(Y, \mathscr{T}_{2}\right)$ are homeomorphic connected nonmetrizable Hausdorff spaces whereas $\left(Y, \mathscr{T}_{1} \vee \mathscr{T}_{2}\right)$ is not connected, and in fact $\left(Y, \mathscr{T}_{1} \vee \mathscr{T}_{2}\right)$ is homeomorphic to a connected subset of the plane together with an isolated point.

In view of the partial order structure on the mobs we are studying, the characterization of a semitree as a certain kind of partially ordered set is the most convenient for the purposes of this paper. Thus a semitree is a partially ordered set $(X, \leqq)$ that satisfies:

(1) There is a least element.

(2) The partial order $\leqq$ is order dense.

(3) Each nonempty subset of $X$ has an infimum in $X$.

(4) For all $x \in X, L(x)$ is a chain.

(5) Each nonempty chain in $X$ has a supremum in.$X$.

If $X$ is an idempotent commutative monotone mob that satisfies condition 0 and has a zero, then $(X, \leqq)$ satisfies (1) through (4) and the following weaker version of (5).

(5') $X$ is conditionally complete.

However, we also wish to bring the topology on $X$ into consideration, and this can be most expeditiously done via a concept of Wolk (1958).

Let $(X, \leqq)$ be a partially ordered set. The interval topology $\mathscr{I}$ is the smallest topology on $X$ for which $L(x)$ and $M(x)$ are closed for all $x \in X$. A topology $\mathscr{T}$ for $X$ is order compatible if and only if for all $x \in X, L(x)$ and $M(x)$ are closed and $\mathscr{T}$ restricted to $L(x)$ equals $\mathscr{I}$ restricted to $L(x)$. A topology $\mathscr{T}$ for $X$ is strongly order compatible if and only if $\mathscr{T}$ is order compatible and $M(x)-\{x\}$ is open for all $x \in X$.

We showed in another paper (1975) that the above definition of order compatible topology agrees with that of Wolk (1958) at least in a semitree. We also gave several examples to illustrate the complexity of the spectrum of order compatible topologies on a semitree. The topology in Example 1 is $\mathscr{I}$. The topology in Example 2 strictly contains $\mathscr{I}$, and so it is not order compatible.

We are now ready to state our first main theorem which easily extends to semitrees. Local connectivity in part (iv) below is slightly more stringent than usual, but the requirements should be clear from the proof.

THEOREM 5. Let $(X, \mathscr{T})$ be an idempotent commutative monotone mob that satisfies condition 0 and has a zero. The following are equivalent. 
(i) $\mathscr{T}$ is strongly order compatible.

(ii) $L(x)$ is compact for all $x \in X$.

(iii) inf $A$, sup $A \in A *$ whenever $A \subset X$ is nonempty and bounded.

(iv) $L(x)$ is locally connected for all $x \in X$.

If, in addition, each chain in $X$ is bounded above, then $(X, \leqq)$ is a semitree.

Proof. The implications (i) $\Rightarrow$ (ii) $\Rightarrow$ (iii) $\Rightarrow$ (iv) follow either from Theorem 4, the proof of Theorem 4, or the observation immediately following Theorem 4. Suppose that (iv) holds and let $x \in X$. Since $\mathscr{I} \subset \mathscr{T}$, every $\mathscr{T}$ connected subset of $L(x)$ is $\mathscr{I}$ connected and hence must be an interval. Thus each $\mathscr{T}$ open set contains an interval about each point, and so $\mathscr{T}=\mathscr{I}$ on $L(x)$. Finally, if each chain is bounded above, then each chain has a supremum. So $(X, \leqq)$ satisfies (5) above and is therefore a semitree.

Conditions (i), (iii), and (iv) in Theorem 5 are equivalent even without the existence of a zero since the proof of Theorem 4 can be suitably modified to handle the zeroless situation.

Corollary 6. 'If $(X, \mathscr{T})$ is an idempotent commutative monotone mob that satisfies condition 0 and has compact maximal chains, then $(X, \leqq)$ is a semitree and $\mathscr{T}$ is a strongly order compatible topology.

COROllary 7. If $X$ is an idempotent commutative monotone mob that satisfies condition 0 and has compact maximal chains, then

(i) $(X, \mathscr{I})$ is compact and

(ii) $X$ has the fixed point property for increasing functions.

Proof. Apply Theorem 6.13 of Muenzenberger and Smithson (1973) and Corollary 6.

It is now most convenient to introduce two concepts used by the authors in other papers. A mod is a semilattice $(X, \leqq)$ in which properties (2), (4), and (5') hold. In other words, a mod is just a semitree perhaps with some endpoints erased. As observed previously, the mobs considered in Theorems 4 and 5 admit the structure of mods with zero, and we now head toward converses of Theorem 5 and Corollary 6.

LEMMA 8. Let $(X$, $\leqq$ ) be a mod. If $\mathscr{T}$ is an order compatible topology for $X$, then $L(x)$ and $M(x)$ are connected for all $x \in X$. If $\mathscr{T}$ is strongly order compatible, then $(X, \mathscr{T})$ is Hausdorff.

Proof. See Muenzenberger and Smithson (1975).

Let $(X, \leqq)$ be a semilattice and let $y \in X$. A branch $B$ at $y$ is a subset of $M(y)$ that is maximal with respect to:

$$
\text { If } y_{1}, y_{2} \in B-\{y\} \text {, then } y_{1} \wedge y_{2} \in B-\{y\} \text {. }
$$


Remark. If $\mathscr{B}$ is a nest of subsets of $M(y)$ satisf ying the above condition, then $\cup \Re$ also satisfies the condition. So Zorn's Lemma implies that each $z \in M(y)$ is contained in a branch at $y$. Note that each branch at $y$ contains $y$. Branches are powerful tools in semitrees; see Muenzenberger and Smithson (1975). We prove two results in the theory of branches that are necessary for our purposes.

Lemma 9. Let $(X, \leqq)$ be a semilattice in which $L(x)$ is a chain for all $x \in X$. If $B$ is a branch at $y \in X$ and $x \in B-\{y\}$, then $[y, x] \subset B$ and $M(x) \subset B$.

Proof. Let $y_{1} \in(y, x]$ and let $y_{2} \in B-\{y\}$. Then $y_{1} \wedge y_{2} \leqq x \wedge y_{2}$. Now either $y_{1} \leqq x \wedge y_{2}$ or $x \wedge y_{2} \leqq y_{1}$. If $y_{1} \leqq x \wedge y_{2}$, then $y_{1} \leqq y_{2}$ and $y_{1} \wedge y_{2}=y_{1}$. If $x \wedge y_{2} \leqq y_{1}$, then $y_{1} \wedge y_{2}=x \wedge y_{2}$. In either case, $y_{1} \wedge y_{2} \neq y$. Thus $[y, x] \subset B$ by the maximality of $B$, and $M(x) \subset B$ follows by a similar argument.

Lemma 10. Let $(X, \leqq)$ be a mod and let $\mathscr{T}$ be a strongly order compatible topology for $X$. If $B$ is a branch at $y \in X$, then $B$ is closed and connected.

Proof. If $x \notin B$, then we may assume that $x \in M(y)-\{y\}$ since $M(y)$ is closed and $B \subset M(y)$. Pick $z$ so that $y<z<x$. Then $x \in M(z)-\{z\}$ and $B \cap(M(z)-\{z\})=\varnothing$ by Lemma 9 . So $B$ is closed.

Now

$$
B-\{y\}=\cup\{M(z): z \in B-\{y\}\}=\cup\{M(z)-\{z\}: z \in B-\{y\}\}
$$

is connected and open. If $y \notin(B-\{y\})^{*}$, then $B-\{y\}$ is clopen, a contradiction. So $B=(B-\{y\})^{*}$ is connected.

The operation $\wedge$ on a semitree is commutative and idempotent, but $\wedge$ need not in general be continuous.

Examples. (4) For $n$ a natural number, let $Y_{n}$ equal the union of the segment from $\left(1 / 2^{2 n}, 0\right)$ to $\left(1 / 2^{2 n}, 1\right)$ and the segment from $\left(1 / 2^{2 n}, 1 / 2\right)$ to $\left(1 / 2^{2 n+1}, 1\right)$ in the plane. Also, let $Y_{-1}=\{(x, 0): 0 \leqq x \leqq 1\}$ and $X=$ $U\left\{Y_{n}: n=-1,0,1, \cdots\right\}$. Define a topology $\mathscr{T}$ for $X$ as follows. Take the usual open sets in $X-\{(0,0)\}$. A basic open set containing $(0,0)$ will consist of the points in $X$ that are to the left of a line $x=c$ and either above a line $y=b$ or below a line $y=a$ where $a, b, c \in(0,1)$ and $a<b$. The basis at $(0,0)$ is obtained by letting $a, b$ and $c$ vary. Let $\leqq$ be the cutpoint order with least element $(1,0)$. Then $(X, \leqq)$ is a semitree, and $\mathscr{T}$ is strongly order compatible. Nonetheless, $\wedge$ is not continuous.

(5) Replace $Y_{n}(n \neq-1)$ in Example 4 by a segment of length one and use the same topology and order. This example shows that an idempotent commutative monotone mob that satisfies condition 0 and has a zero need not be locally connected. 
Consideration of Examples 4 and 5 leads to the following definition. Let $(X, \leqq)$ be a semilattice and let $\mathscr{T}$ be a topology for $X$. Then $\wedge$ is continuous on the diagonal (with respect to $\mathscr{T}$ ) if and only if for each $x \in X$ and each open set $U$ containing $x$, there is an open set $V$ containing $x$ such that $V \wedge V \subset U$.

Remark. Clearly, continuity implies continuity on the diagonal, but the converse is false even for semitrees unless the topology is strongly order compatible. (See Theorem 11 and Corollary 12.) If $(X, \leqq)$ is a semitree, then $\wedge$ is continuous on the diagonal with respect to any strongly order compatible topology $\mathscr{T}$ for which $(X, \mathscr{T})$ is locally connected; see Muenzenberger and Smithson (1975).

THeOREM 11. Let $(X, \leqq)$ be a mod and $\mathscr{T}$ be a strongly order compatible topology for $X$. If $\wedge$ is continuous on the diagonal, then $(X, \mathscr{T}, \wedge)$ is an idempotent commutative monotone mob that satisfies condition 0 .

Proof. Clearly, $\wedge$ is a commutative idempotent operation that satisfies condition 0 . We must show that $\wedge$ is continuous and monotone. To see that $\wedge$ is monotone, note that for $y \in X$

$$
\begin{aligned}
\wedge^{-1}(y) & =(\{y\} \times M(y)) \cup(M(y) \times\{y\}) \cup \cup \mathscr{B} \text { where } \\
\mathscr{B} & =\left\{B \times B^{\prime}: B, B^{\prime} \text { are distinct branches at } y\right\} .
\end{aligned}
$$

For continuity, let $y, z \in X$ and $x=y \wedge z$ and let $W$ be any open set containing $x$. We must construct open sets $U, V$ such that $y \in U, z \in V$, and $U \wedge V \subset W$. The case $y=z$ is exactly continuity on the diagonal. Suppose now that $y$ and $z$ are not comparable. Then pick $y_{1} \in(y \wedge z, y)$ and $z_{1} \in(y \wedge z, z)$ and set $U=M\left(y_{1}\right)-\left\{y_{1}\right\}$ and $V=M\left(z_{1}\right)-\left\{z_{1}\right\}$. Finally, suppose that $z<y$. We may choose $x_{1} \in(x, y)$ to satisfy $\left[x, x_{1}\right] \subset W$ since $\mathscr{T}$ is order compatible. Define $U=M\left(x_{1}\right)-\left\{x_{1}\right\}$. If $x$ is a zero, then set $V=W-M\left(x_{1}\right)$. If $x$ is not a zero, then there is $x_{2}<x$ so that $\left[x_{2}, x\right] \subset W$. In this case, set

$$
V=\left(W \cap\left(M\left(x_{2}\right)-\left\{x_{2}\right\}\right)\right)-M\left(x_{1}\right) .
$$

Thus $\wedge$ is continuous.

CoRollary 12. Let $(X, \leqq)$ be a semitree and let $\mathscr{T}$ be a strongly order compatible topology for $X$. If $\wedge$ is continuous on the diagonal, then $(X, \mathscr{T}, \wedge)$ is an idempotent commutative monotone mob that satisfies condition 0 and has compact maximal chains.

We obtain Ward's Theorem from Corollaries 6 and 12 and results of Muenzenberger and Smithson (1975). 
THEOREM 13. A necessary and sufficient condition that $X$ be a tree is that $X$ be a compact idempotent commutative monotone mob that satisfies condition 0 .

We could obtain many fixed point theorems analogous to Corollary 7 by combining Corollary 6 with the results of Muenzenberger and Smithson (1973, 1974). Rather than write these out explicitly, we close with a conjecture which if true would be a generalization of several results including the Brouwer Fixed Point Theorem.

CONJECTURE. A compact idempotent commutative monotone mob has the fixed point property.

EXAMPLE. (6) The monotone assumption is critical to the worth of the conjecture since Cohen (1974) has given several examples of compact connected topological semilattices without the fixed point property. One can easily see that Cohen's mobs are not monotone by computing $M(1,1,0)$, for example, and evoking Lemma 2. The authors are indebted to the referee for bringing Cohen's paper to their attention.

\section{References}

H. Cohen (1974), 'Compact connected semilattices without the fixed point property', Semigroup Forum 7, 375-379.

T. B. Muenzenberger and R. E. Smithson (1973), 'Fixed point structures', Trans. Amer. Math. Soc. 184, 153-173.

T. B. Muenzenberger and R. E. Smithson (1974), 'Refluent multifunctions on semitrees', Proc. Amer. Math. Soc. 44, 189-195.

T. B. Muenzenberger and R. E. Smithson (1975). 'The structure of nested spaces', Trans. Amer. Math. Soc. 201, 57-87.

L. E. Ward, Jr. (1957), 'Mobs, trees and fixed points', Proc. Amer. Math. Soc. 8, 798-804.

E. S. Wolk (1958), 'Order-compatible topologies on a partially ordered set', Proc. Amer. Math. Soc. 9, 524-529.

Kansas State University

Manhattan, Kansas 66506

U.S.A.

University of Wyoming and University of Houston U.S.A. 